\title{
STUDY OF LARIVICIDAL ACTIVITY OF PHYTOTOXIN FROM LASIOSIPHON ERIOCEPHALUS PLANT AGAINST MOSQUITO AEDES AEGYPTI AND ANOPHELES STEPHENSI
}

\author{
R.G. Patil \\ Emeritus Fellow, P.G. Department of Zoology, \\ L.B.S.College of Arts, Science and Commerce, Satara \\ E. Mail- ramraopatil21@yahoo.com
}

Communicated : 17.12 .18

Accepted : 21.01.18

Published: 30.01 .19

\begin{abstract}
Insecticidal and pharmacological properties are recognized in the plant Lasiosiphon eriocephalus. Different concentrations such as 175, 200, 225,250, 275 and 300 ppm of extract of leaves of plant Lasiosiphon eriocephalus are prepared. The $4^{\text {th }}$ instar larvae of mosquito Aedes aegypti and Anopheles stephensi are exposed to the concentrations $175,200,225,250,275$ and $300 \mathrm{ppm}$ for $48 \mathrm{hrs}$. to study the mortality of larvae for $2,4,8,12,24$ and $48 \mathrm{hrs}$. From the mortality table the values of 'a' and 'b' are calculated and with the help of these values the LC50 values of phytotoxin $L$. eriocephalus to the larvae of mosquito A. egypti are calculated and these LC50 values are observed as $288.63,283.47,240.81,213.07,202.06$ and $184.13 \mathrm{ppm}$ respectively for 2,4,8,12,24 and $48 \mathrm{hrs}$ where as LC50 values of this phytotoxin to the larvae of A. stephensi are observed as 318.71, 240.08, 224.06, $210.14,198.62$ and $187.25 \mathrm{ppm}$ respectively for $2,4,8,12,24$ and $48 \mathrm{hrs}$. There results revealed that phytotoxin from plant $L$. eriocephalus can be used as efficient source in the control of mosquito A. aegypti by destroying the larval stage.
\end{abstract}

Key words :- Phytotoxin , L. eriocephalus, A. aegypti and A. stephensi.

\section{INTRODUCTION:}

Mosquitoes A.aegypti and A. stephensi transmit diseases like dengue and chicken guinea in human being (Ghosh et al., 2012). Hence to prevent the proliferation of mosquito diseases has became vary essential to control the mosquitoes. Use of synthetic insecticides, organophosphates and organochlorides is a common and major practice of human being to control the mosquitoes (Ghosh et al., 2012). But

Due to the concern of environment and human health the most effective alternative is a use of phytotoxin. Because of degradable nature and no effect on non target species. Hence it is used as a sustainable method to control mosquitoes. Different scientists such as Wiseman and Chapagain (2006), Mathew et al., (2009), Patil et al., (2010), Remia and Logaswamy (2010), Ghosh et al., (2012) Yenkanchi et al., (2014) and Mullai and Jebanesan (2017) were studied the potential of plant extracts in the control of mosquito species.

Efforts have made in this work to study the potential of phytotoxin from extract of leaves of $L$. eriocephalus in the control of mosquito A. aegypti and $A$. stephensi.

MATERIALS AND METHOD :

Use of Plant Lasiosiphon eriocephalus (Meissn) Decaisne of family Thymeleaceae are used as a phytotoxin where as $4^{\text {th }}$ instar larvae because of uncontrolled technical and operational practices the use of synthetic chemicals became unsuccessful. Use of synthetic chemicals also not accepted due to high cost of synthetic chemicals, concern of environment, harmful effects on human health and non target population, biodegradable nature and increasing resistance in insects (Brown,1986 and Russel et al., 2009).

of $A$. aegypti and $A$. stephensi are used for the experiment.

\section{a) Preparation of Plant extracts :-}

i. The plants Lasiosiphon eriocephalus identified by expert botanist for their selection.

ii. Matured leaves and fruits of related plant were collected, washed with water and dried at room temperature in a shed.

iii. Dried leaves and fruits of related plant were powdered with mechanical device.

iv. Dried powder was extracted in acetone (100 gm in $300 \mathrm{ml}$ acetone) for 12 to $15 \mathrm{hrs}$ with the help of Soxhlet's apparatus.

v. Solvent powder was evaporated with help of vacuum evaporator and stored in airtight desiccators.

b) Collection of larvae of Aedes aegypti and Anopheles stephensi:-

i. Species of mosquitoes were identified with standard identification keys. 
ii. Larvae were cultured and maintained in the laboratory at $27 \pm 1^{\circ} \mathrm{C}$ and $85 \%$ of relative humidity.

iii. Larval forms were maintained in trays by providing dog biscuits and yeast powder in ratio 3:1

\section{c) Bioassay test :- (WHO, 1981)}

i. Different concentrations of plant extracts from $125 \mathrm{ppm}$ to $300 \mathrm{ppm}$ were prepared in $500 \mathrm{ml}$ beakers. These concentrations were decided after taking pre-test.

ii. Different larval stages (instars) of both species were kept in beakers with different concentrations.

iii. Twenty larvae of each species were exposed to above concentrations in beakers/trays for 2, 4, $8,12,24$ and $48 \mathrm{hrs}$.

iv. Control set was also maintained.

v. Experiment was repeated for five times.

vi. By counting no. of dead larvae percent mortality was calculated with the help of probit analysis method (Fisher and Yates, 1963) for each exposure period.

\section{RESULTS AND DISCUSSION:-}

Mortality study has a key role in the toxicological studies. The potential of the phytotoxin is studied by studying the LC50 of the plant toxin against the target organism. In this study larvae of the $A$. aegypti and $A$. stephensi were exposed to the different concentrations such as 175, 200, 225, 250, 275 and $300 \mathrm{ppm}$. For 2, 4, 8, 12, 24 and $48 \mathrm{hrs}$. of exposure period for the study of larvicidal activity of the phytotoxin.

When larvae of $A$. aegypti and $A$. stephensi were exposed to the concentrations

Table No. 1.1

Numerical data for estimation of 'b' and 'a' relations to plant Lasiosiphon eriocephalus to A. aegypti for different exposure period

\begin{tabular}{|c|c|c|c|c|c|c|c|c|c|c|c|c|c|c|c|c|c|}
\hline \multicolumn{6}{|c|}{$2 \mathrm{hrs}$} & \multicolumn{6}{|c|}{$4 \mathrm{hrs}$} & \multicolumn{6}{|c|}{$8 \mathrm{hrs}$} \\
\hline $\begin{array}{l}\text { Morta } \\
\text { lity \% }\end{array}$ & $\begin{array}{l}\text { Probit } \\
\text { (Y) }\end{array}$ & $\begin{array}{l}\text { Conc in } \\
\operatorname{ppm}(\mathrm{X})\end{array}$ & $\operatorname{LnX}$ & $\operatorname{LnX}^{2}$ & LnXY & $\begin{array}{l}\text { Morta } \\
\text { lity \% }\end{array}$ & $\begin{array}{c}\text { Probit } \\
(\mathrm{Y})\end{array}$ & $\begin{array}{l}\text { Conc. in } \\
\text { ppm (X) }\end{array}$ & $\operatorname{LnX}$ & $n X^{2}$ & $\begin{array}{c}\operatorname{LnX} \\
Y\end{array}$ & \begin{tabular}{|l|} 
mortal \\
ity \%
\end{tabular} & $\begin{array}{c}\text { Probit } \\
(Y)\end{array}$ & $\begin{array}{l}\text { Conc. in } \\
\text { ppm (X) }\end{array}$ & $\operatorname{LnX}$ & $\operatorname{LnX}^{2}$ & $\operatorname{LnXY}$ \\
\hline 00 & -- & 175 & 5.16 & 26.62 & -- & 00 & -- & 75 & 5.16 & 26.62 & 18.32 & 10 & 3.55 & 175 & 5.16 & 26.62 & 18.32 \\
\hline 05 & 3.55 & 200 & 5.30 & 28.09 & 18.82 & 05 & 3.55 & 00 & 5.30 & 28.09 & 16.70 & 20 & 4.16 & 200 & 5.30 & 28.09 & 22.05 \\
\hline 05 & 3.55 & 225 & 5.42 & 29.37 & 19.24 & 10 & 3.72 & 25 & 5.42 & 29.37 & 22.55 & 20 & 4.16 & 225 & 5.42 & 29.37 & 22.55 \\
\hline 20 & 4.15 & 250 & 5.52 & 30.47 & 22.91 & 15 & 3.96 & 50 & 5.52 & 30.47 & 25.45 & 60 & 5.25 & 250 & 5.52 & 30.47 & 28.98 \\
\hline 25 & 4.32 & 275 & 5.62 & 31.58 & 24.28 & 30 & 4.48 & 75 & 5.62 & 31.58 & 27.37 & 80 & 5.84 & 275 & 5.62 & 31.58 & 32.82 \\
\hline \multirow[t]{2}{*}{45} & 4.87 & 300 & 5.70 & 32.49 & 27.76 & 55 & 5.13 & 300 & 5.70 & 32.49 & 30.72 & 100 & -- & 300 & 5.70 & 32.49 & -- \\
\hline & $\begin{array}{l}\Sigma \mathrm{Y}=20 \\
44 \\
\bar{Y}=4.08\end{array}$ & $\begin{array}{l}\text { No.of } \\
\text { conc. } \\
=06\end{array}$ & \begin{tabular}{|l}
$\Sigma \operatorname{LnX}=$ \\
32.72 \\
$\operatorname{Ln} \bar{X}=$ \\
5.45
\end{tabular} & $\begin{array}{l}\Sigma \operatorname{LnX}^{2} \\
=178.62\end{array}$ & $\begin{array}{l}\Sigma \operatorname{LnXY} \\
= \\
113.01\end{array}$ & & \begin{tabular}{|l}
\multicolumn{1}{|c}{$\Sigma \mathrm{Y}=$} \\
20.84 \\
$\overline{\mathrm{Y}}=$ \\
4.17
\end{tabular} & $\begin{array}{l}\text { No.of } \\
\text { conc. } \\
=06\end{array}$ & \begin{tabular}{|l|}
$\Sigma \operatorname{Ln} X$ \\
$=$ \\
32.72 \\
$\operatorname{Ln} \bar{X}$ \\
$=$ \\
5.45 \\
\end{tabular} & $\begin{array}{l}\Sigma \operatorname{Ln} X^{2} \\
= \\
178.62\end{array}$ & \begin{tabular}{|l|}
$\sum \operatorname{LnXY}$ \\
$=$ \\
115.26
\end{tabular} & & $\begin{array}{l}\sum \mathrm{Y}= \\
22.96 \\
\bar{Y}= \\
4.60\end{array}$ & $\begin{array}{l}\text { No.of } \\
\text { conc. }= \\
05\end{array}$ & \begin{tabular}{|l}
$\Sigma \operatorname{Ln} X$ \\
$=$ \\
32.72 \\
$\operatorname{Ln} \bar{X}$ \\
$=$ \\
5.45 \\
\end{tabular} & \begin{tabular}{|l|}
$\Sigma \operatorname{LnX} X^{2}$ \\
$=$ \\
178.62
\end{tabular} & $\begin{array}{l}\operatorname{LnXY} \\
= \\
124.7 \\
2\end{array}$ \\
\hline
\end{tabular}

$175,200,225,250,275$ and 300 ppm for $2,4,8,12,24$ and $48 \mathrm{hrs}$. of exposure period, it is observed that the rate of mortality of larvae of both species increases with increased concentration and time of exposure. Similar type of results were obtained by Choochote (2004) in A. aegypti, Mullai and Jebaneesan (2007) in $C$. quinque fasciatus, Patil et al., (2010), in $A$. aegypti and A. stephensi, Ghosh et al., (2014) in different mosquitoes and Yenkanchi (2014) in A. aegypti.

By using the data of percentage mortality and no. of concentrations the LC50 values were calculated with the help of probit analysis method. LC50 values of plant $L$. eriocephalus to the larvae of $A$. aegypti were found as 288.63, 283.47, 240.81, 213.07, 202.06 and $184.13 \mathrm{ppm}$ respectively for $2,4,8$, 12 , 24, and $48 \mathrm{hrs}$. whereas LC50 values of $L$. eriocephalus to the larvae of $A$. stephensi were found as 318.71, 240.08, 224.06, 210.14, 198.62 and 187.25 respectively for $2,4,8,12,24$ and 40 hrs. (Table No.3) From there observations it can be concluded that the phytotoxin from $L$. eriocephalus is more effective in $A$. aegypti as compared to the A. stephensi.

\section{ACKNOWLEDGEMENT :-}

I am grateful to UGC for awarding me the 'Emeritus Fellowship' because of which I have control. I am also thankful to the Prin. Abhayakumar Salunkhe, President of Shri Swami Vivekanand Shikshan Santha and Prin. R.V.Shejwal for providing me the facilities. worked on this valuable work of mosquito 
Table No. 1.2

Numerical data for estimation of ' $b$ ' and ' $a$ ' relations to plant Lasiosiphon eriocephalus to $A$. aegypti for different exposure period

\begin{tabular}{|c|c|c|c|c|c|c|c|c|c|c|c|c|c|c|c|c|c|}
\hline \multicolumn{6}{|c|}{$12 \mathrm{hrs}$} & \multicolumn{6}{|c|}{24 hrs } & \multicolumn{6}{|c|}{$48 \mathrm{hrs}$} \\
\hline $\begin{array}{l}\text { Mortali } \\
\text { ty } \%\end{array}$ & \begin{tabular}{|l} 
Probit \\
$(\mathrm{Y})$
\end{tabular} & $\begin{array}{l}\text { Conc. in } \\
\text { ppm (X) }\end{array}$ & $\operatorname{Ln} X$ & $\operatorname{Ln} X^{2}$ & LnXY & $\begin{array}{l}\text { Morta } \\
\text { lity \% }\end{array}$ & $\begin{array}{l}\text { Probit } \\
(Y)\end{array}$ & $\begin{array}{l}\text { Conc. in } \\
\operatorname{ppm}(\mathrm{X})\end{array}$ & LnX & $\operatorname{Ln} X^{2}$ & $\operatorname{LnXY}$ & $\begin{array}{l}\text { Mort } \\
\text { ality }\end{array}$ & \begin{tabular}{|l} 
Probit \\
$(\mathrm{Y})$
\end{tabular} & $\begin{array}{l}\text { Conc. in } \\
\text { ppm (X) }\end{array}$ & $\operatorname{Ln} X$ & $\operatorname{Ln} X^{2}$ & $\operatorname{LnXY}$ \\
\hline 20 & 4.16 & 175 & 5.16 & 26.62 & 26.62 & 20 & 4.16 & 175 & 5.16 & 26.62 & 23.12 & 45 & 4.87 & 175 & 5.16 & 26.62 & 25.13 \\
\hline 40 & 4.75 & 200 & 5.30 & 28.09 & 28.09 & 40 & 4.75 & 200 & 5.30 & 28.09 & 25.81 & 55 & 5.13 & 200 & 5.30 & 28.09 & 27.19 \\
\hline 45 & 4.87 & 225 & 5.42 & 29.37 & 29.37 & 55 & 5.13 & 225 & 5.42 & 29.37 & 29.21 & 90 & 6.28 & 225 & 5.42 & 29.37 & 34.04 \\
\hline 85 & 6.04 & 250 & 5.52 & 30.47 & 30.42 & 75 & 5.67 & 250 & 5.52 & 30.47 & 35.60 & 100 & -- & 250 & 5.52 & 30.47 & -- \\
\hline 100 & -- & 275 & 5.62 & 31.58 & 31.58 & 100 & -- & 275 & 5.62 & 31.58 & -- & 100 & -- & 275 & 5.62 & 31.58 & -- \\
\hline \multirow[t]{2}{*}{100} & -- & 300 & 5.70 & 32.49 & 32.49 & 100 & -- & 300 & 5.70 & 32.49 & -- & 100 & -- & 300 & 5.70 & 32.49 & -- \\
\hline & $\begin{array}{l}\Sigma Y= \\
19.82 \\
\bar{Y}=4.96\end{array}$ & $\begin{array}{l}\text { No.of } \\
\text { conc. } \\
=04\end{array}$ & $\begin{array}{l}\sum \operatorname{LnX}= \\
32.72 \\
\operatorname{Ln} \bar{X} \\
=5.45\end{array}$ & $\begin{array}{l}\Sigma \operatorname{LnX}^{2} \\
=178.62\end{array}$ & $\begin{array}{l}\sum \operatorname{LnX} X \\
Y= \\
106.39\end{array}$ & & $\begin{array}{l}\Sigma \mathrm{Y}= \\
20.71 \\
\bar{Y}= \\
.17\end{array}$ & $\begin{array}{l}\text { No.of } \\
\text { conc. } \\
=04\end{array}$ & $\begin{array}{l}\Sigma \operatorname{LnX} \\
= \\
32.72 \\
\operatorname{Ln} \bar{X} \\
=5.45\end{array}$ & \begin{tabular}{|l}
$\Sigma \operatorname{Ln} X^{2}$ \\
$=$ \\
178.62
\end{tabular} & $\begin{array}{l}\sum \operatorname{Ln} X Y \\
=105 . \\
75\end{array}$ & & $\begin{array}{l}\Sigma Y= \\
16.28 \bar{Y}= \\
5.43\end{array}$ & $\begin{array}{l}\text { No.of } \\
\text { conc. } \\
=03\end{array}$ & $\begin{array}{l}\Sigma \operatorname{LnX} \\
= \\
32.72 \\
\operatorname{Ln} \bar{X} \\
= \\
5.45 \\
\end{array}$ & $\begin{array}{l}\Sigma \operatorname{LnX}^{2} \\
=178.62\end{array}$ & $\begin{array}{l}\Sigma \operatorname{LnXY} \\
=86.3 \\
6\end{array}$ \\
\hline
\end{tabular}

Table No. 2.1

Numerical data for estimation of ' $b$ ' and ' $a$ ' relations to plant Lasiosiphon eriocephalus to $A$. stephensi for different exposure period

\begin{tabular}{|c|c|c|c|c|c|c|c|c|c|c|c|c|c|c|c|c|c|}
\hline \multicolumn{6}{|c|}{2 hrs } & \multicolumn{6}{|c|}{4 hrs } & \multicolumn{6}{|c|}{8 hrs } \\
\hline $\begin{array}{l}\text { Mortali } \\
\text { y } \%\end{array}$ & $\begin{array}{l}\text { Probit } \\
(Y)\end{array}$ & $\begin{array}{l}\text { Conc in } \\
\operatorname{ppm}(X)\end{array}$ & $\operatorname{Ln} X$ & $\operatorname{Ln} X^{2}$ & $\operatorname{LnXY}$ & $\begin{array}{l}\text { Mortali } \\
\text { ty \% }\end{array}$ & $\begin{array}{l}\text { Probit } \\
(Y)\end{array}$ & $\begin{array}{l}\text { Conc. in } \\
\text { ppm (X) }\end{array}$ & $\operatorname{Ln} X$ & $\operatorname{Ln} X^{2}$ & LnXY & $\begin{array}{l}\text { Mortali } \\
\text { ty } \%\end{array}$ & $\begin{array}{l}\text { Probit } \\
(Y)\end{array}$ & $\begin{array}{l}\text { Conc. in } \\
\text { ppm (X) }\end{array}$ & $\operatorname{Ln} X$ & $\operatorname{LnX}^{2}$ & $\operatorname{LnXY}$ \\
\hline 0 & -- & 175 & 5.16 & 26.62 & -- & 05 & 3.55 & 175 & 5.16 & 26.62 & 18.32 & 10 & 3.72 & 175 & 5.16 & 26.62 & 19.19 \\
\hline 05 & 3.55 & 200 & 5.30 & 28.09 & 18.81 & 20 & 4.16 & 200 & 5.30 & 28.09 & 22.05 & 35 & 4.61 & 200 & 5.30 & 28.09 & 24.43 \\
\hline 20 & 4.16 & 225 & 5.42 & 29.37 & 22.55 & 20 & 4.16 & 225 & 5.42 & 29.37 & 22.55 & 45 & 4.87 & 225 & 5.42 & 29.37 & 26.39 \\
\hline 25 & 4.33 & 250 & 5.52 & 30.47 & 23.90 & 50 & 5.00 & 250 & 5.52 & 30.47 & 27.60 & 55 & 5.13 & 250 & 5.52 & 30.47 & 28.32 \\
\hline 35 & 4.61 & 275 & 5.62 & 31.58 & 25.91 & 55 & 5.13 & 275 & 5.62 & 31.58 & 28.83 & 95 & 6.44 & 275 & 5.62 & 31.58 & 36.19 \\
\hline \multirow[t]{2}{*}{40} & 4.75 & 300 & 5.70 & 32.49 & 27.07 & 70 & 5.32 & 300 & 5.70 & 32.49 & 30.32 & 100 & -- & 300 & 5.70 & 32.49 & -- \\
\hline & $\begin{array}{l}\mathrm{Y} \\
=21.4 \\
\bar{Y}=4.28\end{array}$ & $\begin{array}{l}\text { No.of } \\
\text { conc. }=0 \\
6\end{array}$ & $\begin{array}{l}\Sigma \operatorname{LnX} \\
= \\
32.72 \\
\operatorname{Ln} \bar{X} \\
= \\
\end{array}$ & $\begin{array}{l}\sum \operatorname{LnX}^{2} \\
= \\
178.62\end{array}$ & $\begin{array}{c}\Sigma \mathrm{LnXY} \\
=11 \\
8.24\end{array}$ & & \begin{tabular}{|l|}
$\Sigma Y$ \\
$=27.32$ \\
$\bar{Y}=$ \\
4.55
\end{tabular} & $\begin{array}{l}\text { No.of } \\
\text { conc. }= \\
06\end{array}$ & $\begin{array}{l}\operatorname{LnX}= \\
32.72 \\
\operatorname{Ln} \bar{X} \\
= \\
5.45 \\
\end{array}$ & 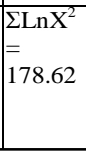 & \begin{tabular}{|l}
$\operatorname{LnXY} X$ \\
$=$ \\
149.6 \\
7
\end{tabular} & & \begin{tabular}{|l}
$\mathrm{Y}$ \\
$=24.7$ \\
7 \\
$\bar{Y}=$ \\
4.95 \\
\end{tabular} & $\begin{array}{l}\text { No.of } \\
\text { conc. } \\
=05\end{array}$ & \begin{tabular}{|l}
$\Sigma \operatorname{LnX} X$ \\
$=$ \\
32.72 \\
$\operatorname{Ln} \bar{X}$ \\
$=$ \\
\end{tabular} & $\begin{array}{l}\begin{array}{l}\sum \operatorname{LnX}^{2} \\
= \\
178.62\end{array} \\
\end{array}$ & $\begin{array}{l}\Sigma \operatorname{LnX} \\
Y= \\
160.3 \\
5\end{array}$ \\
\hline
\end{tabular}

Table No. 2.2

Numerical data for estimation of ' $b$ ' and ' $a$ ' relations to plant Lasiosiphon eriocephalus to $A$. stephensi for different exposure period

\begin{tabular}{|c|c|c|c|c|c|c|c|c|c|c|c|c|c|c|c|c|c|}
\hline \multicolumn{6}{|c|}{$12 \mathrm{hrs}$} & \multicolumn{6}{|c|}{24 hrs } & \multicolumn{6}{|c|}{$48 \mathrm{hrs}$} \\
\hline $\begin{array}{l}\text { Mortali } \\
\text { ty } \%\end{array}$ & $\begin{array}{l}\text { Probit } \\
\text { (Y) }\end{array}$ & $\begin{array}{l}\text { Conc. in } \\
\operatorname{ppm}(\mathrm{X})\end{array}$ & $\operatorname{Ln} X$ & $\operatorname{Ln} X^{2}$ & $\operatorname{LnXY}$ & $\begin{array}{l}\text { Mortali } \\
\text { ty \% }\end{array}$ & $\begin{array}{l}\text { Probit } \\
\text { (Y) }\end{array}$ & $\begin{array}{l}\text { Conc. in } \\
\text { ppm (X) }\end{array}$ & LnX & $\operatorname{Ln} X^{2}$ & $\operatorname{LnXY}$ & $\begin{array}{l}\text { Morta } \\
\text { lity \% }\end{array}$ & $\begin{array}{l}\text { Probit } \\
\text { (Y) }\end{array}$ & $\begin{array}{l}\text { Conc. in } \\
\text { ppm (X) }\end{array}$ & LnX & $\operatorname{LnX}^{2}$ & LnXY \\
\hline 25 & 4.33 & 175 & 5.16 & 26.62 & 22.34 & 30 & 4.48 & 175 & 5.16 & 26.62 & 23.12 & 40 & 4.87 & 175 & 5.16 & 26.62 & 25.13 \\
\hline 40 & 4.55 & 200 & 5.30 & 28.09 & 24.11 & 45 & 4.87 & 200 & 5.30 & 28.09 & 25.81 & 55 & 5.39 & 200 & 5.30 & 28.09 & 28.57 \\
\hline 55 & 5.13 & 225 & 5.42 & 29.37 & 27.80 & 65 & 5.67 & 225 & 5.42 & 29.37 & 30.73 & 95 & -- & 225 & 5.42 & 29.37 & -- \\
\hline 80 & 5.84 & 250 & 5.52 & 30.47 & 32.24 & 95 & -- & 250 & 5.52 & 30.47 & -- & 100 & -- & 250 & 5.52 & 30.47 & -- \\
\hline 100 & -- & 275 & 5.62 & 31.58 & -- & 100 & -- & 275 & 5.62 & 31.58 & -- & 100 & -- & 275 & 5.62 & 31.58 & -- \\
\hline \multirow[t]{2}{*}{100} & -- & 300 & 5.70 & 32.49 & -- & 100 & -- & 300 & 5.70 & 32.49 & -- & 100 & -- & 300 & 5.70 & 32.49 & -- \\
\hline & \begin{tabular}{|l|}
$\Sigma \mathrm{Y}$ \\
$=19.85$ \\
$\overline{\mathrm{Y}}=4.96$
\end{tabular} & $\begin{array}{l}\text { No.of } \\
\text { conc. }=0 \\
4\end{array}$ & $\begin{array}{l}\sum \operatorname{LnX} \\
= \\
32.72 \\
\operatorname{Ln} \bar{X}\end{array}$ & $\begin{array}{l}\sum \operatorname{LnX}^{2} \\
= \\
178.62\end{array}$ & $\begin{array}{l}\sum \operatorname{LnX} \\
Y= \\
106.4 \\
9\end{array}$ & & $\begin{array}{l}\bar{Y} Y \\
=15.02 \\
\bar{Y}=5.01\end{array}$ & $\begin{array}{l}\text { No.of } \\
\text { conc. }= \\
03\end{array}$ & $\begin{array}{l}\sum \operatorname{LnX} \\
= \\
32.72 \\
\operatorname{Ln} \bar{X}\end{array}$ & $\begin{array}{l}\sum \operatorname{LnX} X^{2} \\
= \\
178.62\end{array}$ & $\begin{array}{l}\Sigma \operatorname{LnXY} \\
= \\
79.66\end{array}$ & & \begin{tabular}{|l|}
$\Sigma Y=$ \\
10.26 \\
$\bar{Y}=5.13$
\end{tabular} & $\begin{array}{l}\text { No.of } \\
\text { conc.= } \\
02\end{array}$ & $\begin{array}{l}\Sigma \operatorname{LnX} \\
= \\
32.72 \\
\operatorname{Ln} \bar{X}\end{array}$ & $\begin{array}{l}\Sigma \operatorname{LnX}^{2} \\
= \\
178.62\end{array}$ & $\begin{array}{l}\Sigma \operatorname{LnXY} \\
= \\
53.7\end{array}$ \\
\hline
\end{tabular}


Table No. 3.1

LC50 values of phytotoxin $L$. eriocephalus to the mosquito larvae of $A$. aegypti and $A$. stephensi for different exposure periods.

\begin{tabular}{|l|l|c|}
\hline \multirow{3}{*}{$\begin{array}{l}\text { Mosquito } \\
\text { larvae }\end{array}$} & $\begin{array}{l}\text { Time of } \\
\text { Exposur } \\
\text { e }\end{array}$ & $\begin{array}{l}\mathrm{LC}_{50} \text { values of phytotoxins in } \\
\text { relation to the } 4^{\text {th }} \text { instar } \\
\text { mosquito larvae }(\mathrm{ppm})\end{array}$ \\
\cline { 2 - 3 } & 2 & L. eriocephalus \\
\hline \multirow{4}{*}{ A. aegypti } & 4 & 288.63 \\
\cline { 2 - 3 } & 8 & 283.47 \\
\cline { 2 - 3 } & 12 & 240.81 \\
\cline { 2 - 3 } & 24 & 213.07 \\
\cline { 2 - 3 } & 48 & 202.06 \\
\hline & 2 & 184.13 \\
\cline { 2 - 3 } & 4 & 318.71 \\
\cline { 2 - 3 } & 8 & 240.08 \\
\cline { 2 - 3 } & 12 & 224.06 \\
\cline { 2 - 3 } & 24 & 210.14 \\
\cline { 2 - 3 } & 48 & 198.62 \\
\hline
\end{tabular}

\section{REFERENCES:-}

i. Abbott WS. (1925) : A method of computing the effectiveness of an insecticide. J Econ Entomol, 18, 265-267.

ii. Brown A.W. (1986) : Insecticide resistance in mosquitoes :a a pragmatic review $J$. Am Mosquito Control Association, 2, 123-140.

iii. Choochote, W., Kanjanapothi, B.T.D., Rattanachanpichai. E., Chaithong, U., Chaiwong, P., Riyong, D. and Pitasawat, B. (2004) : Potential of crude seed extract of celery, Apium graveolens L., against the mosquito Aedes aegypti (1.). Journal of Vector Ecology 12, 340-346.

ii. Fisher R.A. and Yates F. (1963) : Statistical tables for biological, agricultural and medical research. Oliver and Byod Edinburgh Tweeddale Court. 68-70.

iii. Ghosh Anupam, Chowdhury Nandita and Chandra Goutam (2012) : Plant extracts as potential mosquito larvicides, Indian J. Med. Res.,135,581-598.

iv. Mathew N., Anita, M. G., Bala, T. S. L., Sivakumar, S. M., Narmada, R. and Kalyanasundaram, M (2009): Larvicidal activity of
Saracaindica, Nyctanthes arbor-trust's, and Clitoria term area extracts against three mosquito vector species. Parasitology Research, 104, 1017-1025.

v. Mullai, K. and Jebanesan, A. (2007) : Larvicidal, ovicidal and repellent activities of the leaf extracts of two cucurbitacious plants against filarial vector Culex quinquefasciatus (Say) (Diptera : Culicidae). Tropical Bio-medicine, 24 (1), 1-6.

vi. Patil, S. V., Patil, C. D., Salunkhe, R. B. and Salunke, B. K. (2010): Larvicidalactivities of six plant extracts against two mosquito species, Aedesaegyptiand Anopheles Stephens. Tropical Biomedicine 27 (3), 360-365.

vii. Wiseman, $Z$ and Chapagain, B. P. (2006) Larvicidal activity of saponin containing extracts and fractions of fruit mesocarp of Balanitesaegyptiaca. Fitoterapia 77:420-424 $4^{\text {th }}$.

viii. Yankanchi S. R., Yadav O. V., and Jadhav G. S. (2014) : Synergistic and individual efficacy of certain plant extracts against dengue vector mosquito, Aedesaegypti. J. Hippest, 7 (1) 22-28. 